TITLE:

\title{
Emission color tunable light- emitting diodes composed of InGaN multifacet quantum wells
}

$\operatorname{AUTHOR}(S):$

Funato, M; Kawakami, Y

CITATION:

Funato, M ... [et al]. Emission color tunable light-emitting diodes composed of InGaN multifacet quantum wells. APPLIED PHYSICS LETTERS 2008, 93(2): 021126.

ISSUE DATE:

2008-07-14

URL:

http://hdl.handle.net/2433/84581

RIGHT:

Copyright 2008 American Institute of Physics. This article may be downloaded for personal use only. Any other use requires prior permission of the author and the American Institute of Physics. 


\title{
Emission color tunable light-emitting diodes composed of InGaN multifacet quantum wells
}

\author{
M. Funato, ${ }^{1, a)}$ K. Hayashi, ${ }^{1}$ M. Ueda,${ }^{1}$ Y. Kawakami, ${ }^{1}$ Y. Narukawa,${ }^{2}$ and T. Mukai ${ }^{2}$ \\ ${ }^{1}$ Department of Electronic Science and Engineering, Kyoto University, Kyoto 615-8510, Japan \\ ${ }^{2}$ Nitride Semiconductor Research Laboratory, Nichia Corporation, Tokushima 774-8601, Japan
}

(Received 5 March 2008; accepted 18 June 2008; published online 18 July 2008)

\begin{abstract}
We demonstrate that the apparent emission colors of InGaN-based light-emitting diodes using microstructured multifacet quantum wells as active layers can externally be controlled over a wide spectral range that encompasses green to blue or white at a color temperature of $4000 \mathrm{~K}$ to blue along the Planckian locus. The controllability relies on facet-dependent polychromatic emissions. The pulsed current operation with the appropriate duties varied their relative intensities and the consequent apparent colors without seriously affecting the total number of emitted photons, particularly for the blue to green variation. (C) 2008 American Institute of Physics. [DOI: $10.1063 / 1.2956404]$
\end{abstract}

A current technological target in lighting is to achieve high color rendering properties without degrading emission efficiency. Although it is generally difficult to simultaneously satisfy both conditions, several manufacturers have recently announced fluorescent lamps with an average color rendering index of $>80$ and a luminous efficiency of $>80 \mathrm{~lm} / \mathrm{W}$, which predict that the technological goal is within a grasp. Then one of the next targets for advanced lighting would be external controllability of the emission color. We suggest, for example, general lighting in which the apparent emission color can be tuned from daylight white to warm white just by rotating a knob, depending on the situation.

Compact solid-state lighting based on semiconductor light-emitting diodes (LEDs) features high emission quantum efficiencies, long lifetime, solidness, and harmlessness compared to conventional fluorescent lamps, and thus, has already impacted various fields such as general lighting and displays. ${ }^{1}$ However, because the bandgap of the active region in an LED inherently dictates the emission wavelength, the output color is nearly monochromatic and, in principle, is difficult to externally control, which is why current white LEDs use a yellow phosphor ${ }^{2-5}$ and full-color displays consist of individual blue, green, and red LEDs. On the other hand, there have been successful attempts at controlling the emission color externally. ${ }^{6,7}$ Reference 6 proposes a threeterminal configuration in which two electrically independent InGaN LEDs emitting at 470 and $535 \mathrm{~nm}$ are vertically connected via a tunneling junction, while Ref. 7 relies on the quantum confinement Stark effect in a commercial, monochromatic InGaN green LED. However, the former requires a complicated device structure, and the latter can achieve only a small variation in the emission wavelength. Herein we demonstrate that proof-of-concept LEDs composed of microstructured $\mathrm{InGaN} / \mathrm{GaN}$ quantum wells (QWs) exhibit polychromatic emission and that a pulse operation enables the apparent emission color to be largely controlled without considerably changing the number of output photons.

It has been reported that regrowth of $\mathrm{GaN}$ on patterned $\mathrm{SiO}_{2}$ masks creates three-dimensional microstructures, which consist of several facets such as (0001), $\{11 \overline{2} 2\}$, and

\footnotetext{
${ }^{a)}$ Electronic mail: funato@kuee.kyoto-u.ac.jp.
}

$\{11 \overline{2} 0\} .{ }^{8,9}$ These results have inspired us to fabricate InGaN/GaN QWs on such microfacets, ${ }^{10-13}$ and very recently, we demonstrated microfacet InGaN LEDs that exhibit polychromatic emission including white due to the facetdependent InGaN well widths and In compositions. ${ }^{13}$ The multifacet LEDs used in this study have essentially the same structure, and consist of underlying three-dimensional $n$-GaN, three-period $\mathrm{InGaN} / \mathrm{GaN}$ QWs as the light emitting layer, and a $p$-GaN cap layer, which is schematically depicted in Fig. 1(a). These LEDs were formed by metalorganic vapor phase epitaxy on $\mathrm{SiO}_{2}$ mask stripes along the

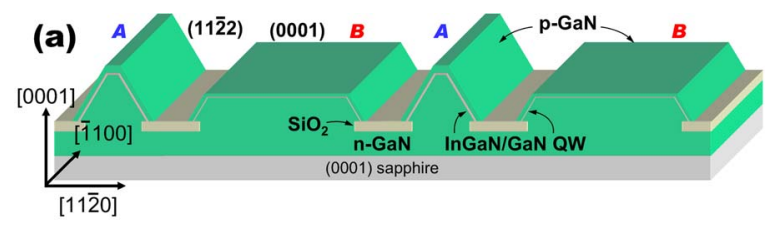

(b)
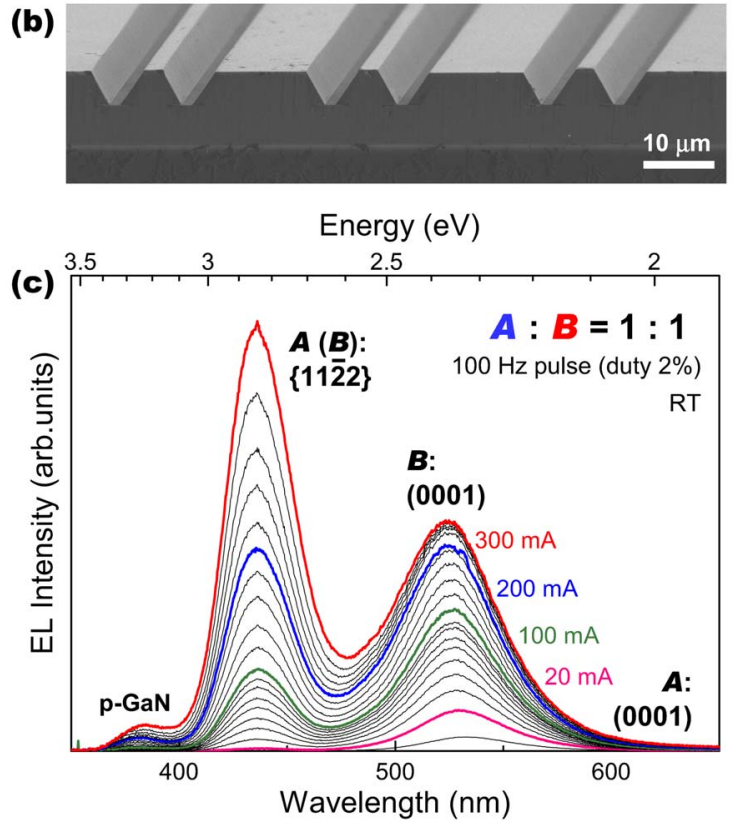

FIG. 1. (Color online) (a) Schematic of a multifacet LED, (b) SEM image of an as-grown multifacet LED and (c) its EL spectra under various injection currents. 


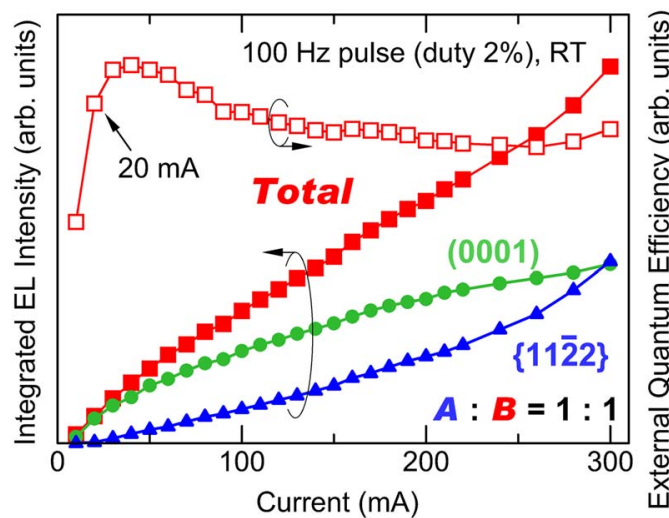

FIG. 2. (Color online) Integrated EL intensity as a function of injection current for the multifacet LED with a pattern mixture of $\boldsymbol{A}: \boldsymbol{B}=1: 1$. Total $\mathrm{EQE}$ is also shown.

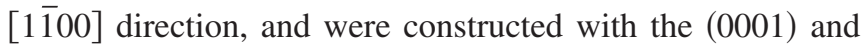
$\{11 \overline{2} 2\}$ facets. (The details of the growth and device structures can be found in Refs. 11-13.) Figure 1(b) shows a sample image of cross sectional scanning electron microscopy (SEM) for an as-grown multifacet LED. To strengthen the emission color controllability, two different structures with a narrow trapezoidal cross section like $\boldsymbol{A}$ and a wide trapezoidal cross section like $\boldsymbol{B}$ were involved within one LED chip. ${ }^{12,13}$ The new period, which was composed of $m$ periods of $A$ and $n$ periods of $B$, is denoted as $\boldsymbol{A}: \boldsymbol{B}=m: n$. Particularly in Figs. 1(a) and 1(b), $\boldsymbol{A}: \boldsymbol{B}=1: 1$. The dimensions of $\boldsymbol{A}$ and $\boldsymbol{B}$ were determined by the $\mathrm{SiO}_{2}$ mask geometry. The opening was $\sim 5 \mu \mathrm{m}$ for $\boldsymbol{A}$ and $\sim 15 \mu \mathrm{m}$ for $\boldsymbol{B}$, while both had a mask stripe $\sim 5 \mu \mathrm{m}$ wide. The LED chip size was $500 \times 500 \mu \mathrm{m}^{2}$, which is larger than conventional LEDs. This larger size was chosen for the reason described below. The LEDs were on wafer without packages. The electroluminescence (EL) spectra were acquired at room temperature under the $100 \mathrm{~Hz}$ pulse operation.

Figure 1(c) shows variations in the EL spectra of the multifacet LED due to injection currents. The duty of the injection pulses was $2 \%$. The spectra clearly demonstrate polychromatic emissions, and their origins were identified from the EL spectra of LEDs without a pattern mixture. The emission at $\sim 435 \mathrm{~nm}$ (blue) was due mainly to the $\{11 \overline{2} 2\}$ QWs in $\boldsymbol{A}$ and partially to those in $\boldsymbol{B}$, whereas the emission at $\sim 525 \mathrm{~nm}$ (yellowish green) was due to the (0001) QWs in $\boldsymbol{B}$, while a broad, weak emission due to the (0001) QWs in $\boldsymbol{A}$ was observed at $\sim 620 \mathrm{~nm}$ (reddish orange). The emission at $380 \mathrm{~nm}$ was from $p$-GaN, which suggests that electrons overflow from $n$-GaN into $p$-GaN due to the absence of an electron blocking $p$-AlGaN layer. The EL spectra under low currents were dominated by the green emission at $\sim 525 \mathrm{~nm}$ from the (0001) QWs in $\boldsymbol{B}$. As the injection current increased, the blue emission from the $\{11 \overline{2} 2\}$ QWs appeared at $\sim 435 \mathrm{~nm}$, and its intensity exceeded that of the (0001) QWs in $\boldsymbol{B}$ near $200 \mathrm{~mA}$.

Figure 2 quantitatively summarizes these observations where the integrated EL intensities are plotted as a function of the injection current. For each facet LED, the integrated EL was extracted by decomposing the total EL spectra with two Gaussian curves. Figure 2 shows that the EL intensity of the (0001) LEDs increases sublinearly, while that of the $\{11 \overline{2} 2\}$ LEDs increases superlinearly. Considering the effi- ciency droop as the injection current increases, which is a ubiquitous characteristic of current InGaN-based LEDs, ${ }^{5}$ the superlinear increase in the EL intensity of the $\{11 \overline{2} 2\}$ LEDs can be interpreted by the variation in the current pass. Under low current, the current through the $\{11 \overline{2} 2\}$ facets is smaller than that of the (0001) facets, which is supported by the negligible EL from the $\{11 \overline{2} 2\}$ LEDs below $20 \mathrm{~mA}$, as shown in Fig. 1(c). Then as the injection current increases, the current through the $\{11 \overline{2} 2\}$ facets superlinearly increases with respect to that of the (0001) facets. This can happen when the turn-on voltage is larger and the series resistance is smaller for the $\{11 \overline{2} 2\}$ LEDs, which implies a higher hole concentration in the $\{11 \overline{2} 2\}$ LEDs. The change of the current pass from the (0001) LEDs emitting green to the $\{11 \overline{2} 2\}$ LEDs emitting blue may be a positive factor for improving the total quantum efficiency because a blue emission generally exhibits a higher quantum efficiency than a green emission. ${ }^{5,14}$ On the one hand, a negative factor is the efficiency droop phenomenon. As a result of competition between those positive and negative factors, the overall external quantum efficiency (EQE) of this multifacet LED, which was extracted by dividing the total integrated EL intensity with the injection current, shows a weak dependency on the injection current, particularly above $100 \mathrm{~mA}$, as shown in Fig. 2. (Quantitative evaluations of EQE have not been performed yet because the current multifacet LEDs are in the demonstration stage, and the device structures have to be optimized by, for example, inserting an electron blocking layer, to extract a full performance.)

The strong injection-current dependence of the EL spectra may be a drawback in terms of the emission color stability, but this can be used to externally control the emission color without changing the EL intensity. The basic idea is as follows. We chose $20 \mathrm{~mA}$ as the standard injection current and $20 \mathrm{~mA} \mathrm{dc}$ current was applied to the LED. Then, the pulse width $t$ was decreased when the injection current $I$ increased in order to maintain a constant number of injection carriers; that is, $I \times t=20 \mathrm{~mA} / 100 \mathrm{~Hz}$. As shown in Fig. 2, the variation of EQEs above $20 \mathrm{~mA}$ is within $\pm 10 \%$ from the standard $(20 \mathrm{~mA})$, and therefore, this manner of the carrier injection may not seriously affect the emission intensity, but may realize various emission colors. Figure 3(a) schematically illustrates the injection pulses. The results of the EL measurements are summarized in Figs. 3(b) and 3(c), where Fig. 3(b) plots the Commission Internationale de I'Éclairage (CIE) 1931 chromaticity diagram, while Fig. 3(c) shows example photographs of a multifacet LED under various injection currents. The emission color continuously changed from yellowish green $(20 \mathrm{~mA})$ to blue $(300 \mathrm{~mA})$ according to the injection current. Moreover, intermediate pastel colors, which cannot be realized by conventional monochromatic LEDs, were obtained. It is noteworthy that the photographs were taken with the same exposure time, indicating that the emission intensity barely changed, but the emission color drastically changed. The adopted pulse frequency of $100 \mathrm{~Hz}$ is sufficiently fast so that human eyes do not see light flicker.

One reason why a large chip, $500 \times 500 \mu \mathrm{m}^{2}$, was used was to achieve a wide range of emission color variation with a reasonable output photon flux. The standard of $20 \mathrm{~mA}$ in this study was determined referring to that of conventional to AIP license or copyright; see http://apl.aip.org/apl/copyright.jsp 


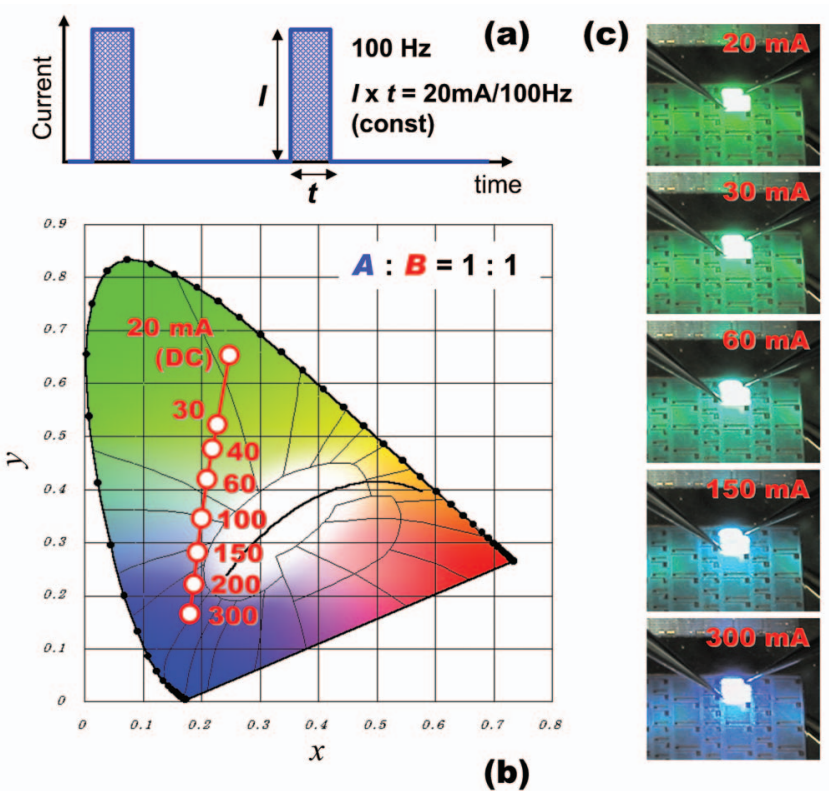

FIG. 3. (Color) (a) Schematic of injection current pulses. Number of injected carriers is constant, regardless of the current values. (b) CIE chromaticity diagram for the EL spectra of the multifacet LED obtained under the pulse operation shown in (a), and (c) example photographs of the multifacet LED emitting various colors.

InGaN-based LEDs with a typical chip size of $~ 300$ $\times 300 \mu \mathrm{m}^{2}$. Therefore, the brightness of the proposed LED is, at least, comparable to, but is expectedly better than that of the conventional (smaller) LEDs because the efficiency droop is suppressed due to lower current densities. ${ }^{3-5}$ As for the emission color variation, we confirmed that a smaller multifacet LED $\left(300 \times 300 \mu \mathrm{m}^{2}\right)$ realized a variation only from $(0.21,0.44)$ for $20 \mathrm{~mA}$ to $(0.17,0.12)$ for $130 \mathrm{~mA}$ on the CIE diagram under the standard driving condition where $20 \mathrm{~mA} / 100 \mathrm{~Hz}$ is constant. Particularly at low currents, the EL spectra could not achieve a monochromatic color, unlike a larger multifacet LED [Fig. 3(b)]. This is simply because the EL spectra are determined not by current, but by current density. That is, to achieve a wide variation of the emission color by a small LED, the driving current and the consequent output photon number must be reduced, which is not desirable for practical uses.

Finally, we like to show primary results for multifacet white LEDs. This white LED had a different structure of $\boldsymbol{A}: \boldsymbol{B}=2: 1$, and the InGaN QW region was fabricated at a slightly lower growth temperature. The EL spectra were quite similar to those of the previous LED, but the lowered growth temperature pushed the emission peaks to longer wavelengths of $\sim 455 \mathrm{~nm}$ (blue) for the $\{11 \overline{2} 2\}$ QWs, 550-560 nm (yellow green) for the (0001) QWs in $\boldsymbol{B}$, and $\sim 700 \mathrm{~nm}$ (red) for the (0001) QWs in $\boldsymbol{A}$. Figure 4(a) plots the CIE chromaticity diagram. The standard current was set to $5 \mathrm{~mA}$ and the injection current was increased in the same manner as above. Interestingly, the emission color was white at a color temperature of $4000 \mathrm{~K}$ for $5 \mathrm{~mA}$, and nearly followed the Planckian locus as the injection current increased. Figure 4(b) displays photographs of LEDs emitting white at a color temperature of $5000 \mathrm{~K}(5.5 \mathrm{~mA})$ or $20000 \mathrm{~K}$ (10 $\mathrm{mA})$. In fact, the apparent color looked pure white at $5000 \mathrm{~K}$, but was bluish white at $20000 \mathrm{~K}$. A remaining issue (a)

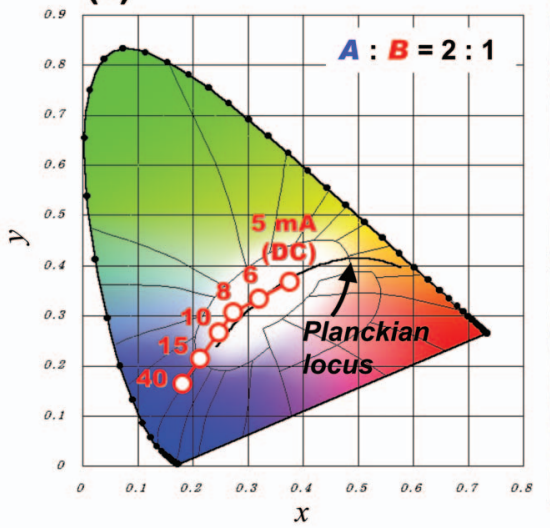

(b)

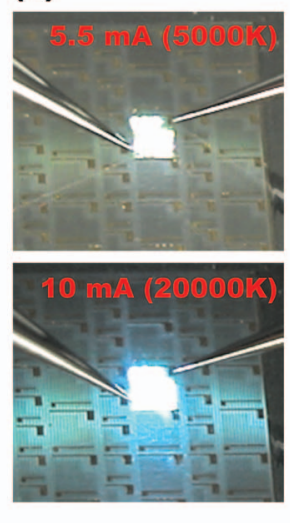

FIG. 4. (Color) (a) CIE chromaticity diagram for the EL spectra of another multicafet white LED obtained under a constant-carrier injection, and (b) example photographs of the LED emitting white at 5000 or $20000 \mathrm{~K}$. The pattern mixture was $\boldsymbol{A}: \boldsymbol{B}=2: 1$ for this white LED.

for this white LED is the inferior quantum efficiency of the yellow-green emission from the (0001) QWs in $\boldsymbol{B}$. Due to this issue, the integrated EL intensity was weak under low injection currents, but considerably increased as the current increased, although the number of injected carriers was constant. Another device architecture to improve the performance is currently under investigation.

To summarize, we demonstrated external control of the apparent emission color of LEDs where InGaN multifacet QWs play a principal role. The pulse operation with the appropriate duties is proposed to tune the EL color without changing the EL intensity. It is noteworthy that such external control of the emission color is quite difficult not only for solid-state lighting, but also for conventional fluorescent lamps and incandescent bulbs. Therefore, we believe that the multifacet LEDs can be a milestone toward next-generation lighting.

${ }^{1}$ J. Y. Tsao, IEEE Circuits Devices Mag. 20, 28 (2004).

${ }^{2}$ K. Bando, K. Sakano, Y. Noguchi, and Y. Shimizu, J. Light Visual Environ. 22, 2 (1998).

${ }^{3}$ Y. Narukawa, J. Narita, T. Sakamoto, K. Deguchi, T. Yamada, and T. Mukai, Jpn. J. Appl. Phys., Part 2 45, L1084 (2006).

${ }^{4}$ Y. Narukawa, M. Sano, M. Ichikawa, S. Minato, T. Sakamoto, T. Yamada, and T. Mukai, Jpn. J. Appl. Phys., Part 2 46, L963 (2007).

${ }^{5}$ M. R. Krames, O. B. Shchekin, R. Mueller-Mach, G. O. Mueller, L. Zhou, G. Harbers, and M. G. Craford, J. Disp. Technol. 3, 160 (2007).

${ }^{6}$ I. Ozden, E. Makarona, A. V. Nurmikko, T. Takeuchi, and M. Krames, Appl. Phys. Lett. 79, 2532 (2001).

${ }^{7}$ T. Azuhata, T. Homma, Y. Ishikawa, and S. F. Chichibu, Jpn. J. Appl. Phys., Part 2 42, L497 (2003).

${ }^{8}$ H. Marchand, J. P. Ibbetson, P. T. Fini, S. Keller, S. P. DenBaars, J. S. Speck, and U. K. Mishra, J. Cryst. Growth 195, 328 (1998).

${ }^{9}$ K. Hiramatsu, K. Nishiyama, M. Onishi, H. Mizutani, M. Narukawa, A. Motogaito, H. Miyake, Y. Iechika, and T. Maeda, J. Cryst. Growth 221, 316 (2000).

${ }^{10}$ K. Nishizuka, M. Funato, Y. Kawakami, Sg. Fujita, Y. Narukawa, and T. Mukai, Appl. Phys. Lett. 85, 3122 (2004).

${ }^{11}$ M. Funato, T. Kotani, T. Kondou, Y. Kawakami, Y. Narukawa, and T. Mukai, Appl. Phys. Lett. 88, 261920 (2006).

${ }^{12}$ M. Ueda, T. Kondou, K. Hayashi, M. Funato, Y. Kawakami, Y. Narukawa, and T. Mukai, Appl. Phys. Lett. 90, 171907 (2007).

${ }^{13}$ M. Funato, T. Kondou, K. Hayashi, S. Nishiura, M. Ueda, Y. Kawakami, Y. Narukawa, and T. Mukai, Appl. Phys. Express 1, 011106 (2008).

${ }^{14}$ T. Mukai, M. Yamada, and S. Nakamura, Jpn. J. Appl. Phys., Part 138 , 3976 (1999). 